appearance, with earlier and more regular vascularization. It showed a higher tensile strength throughout testing. Re-epithelization was accelerated.

Key words: Anastomosis, intestinal - Technique, suture - Microangiography - Histology.

Zusammenfassung. An 104 Anastomosen des Hunde-Magendarmtraktes wurden ein- und zweireihig invertierende und einreihig auf StoB adaptierende Nahttechniken 4, 8 und 18 Tage p.O. makroskopisch, im Zugversuch, mikroangiographisch und histologisch untersucht. Bei allen Kriterien erwies sich die schichtgerechte Anastomose überlegen; sie heilte ohne Wulstbildung oder Stenosierung, zeigte während des gesamten Verlaufes höhere mechanische Belastbarkeit und bildet unter früherem und geordneterem Gefäßdurchbau sowie frühzeitiger Reepithelisierung eine eng-umschriebene zarte Narbe.

Schliisselwörter: Darmanastomose - Nahttechnik - Mikroangiographie - Histologie.

\title{
141. Der Billroth-I-Magen: funktionelle und morphologische Ergebnisse
}

\author{
B. Werner, M. Rehner und N. Soehendra \\ Chirurgische Universitätsklinik Hamburg
}

\section{Morphology and Pathophysiology of the Billroth-I Gastroduodenostomy}

Summary. Endoscopy and biopsy, X-rays, and acid-secretion studies were undertaken in 92 patients who had undergone partial gastric resection (gastroduodenostomy, Billroth-I) 1-7 years previously. In addition, the amount of duodenogastric reflux was evaluated by measuring bromsulphalein excretion in the gastric juice. The following results were found: (1) the severity of duodenal gastric reflux were decisive in the development of atrophic mucosal changes; (2) the acid secretion was reduced by 87 percent against its preoperative level; (3) there is a special motility pattern peculiar to the gastric stump, which-if present-makes normal emptying possible.

Key words: Gastroduodenostomy - Gastritis, reflux - Motility, gastric stump.

Zusammenfassung. 92 nach Billroth-I magenresezierte Patienten wurden 1-7 Jahre p.o. endoskopisch-bioptisch, röntgenologisch und säuresekretionsanalytisch untersucht. Der duodenogastrische Reflux wurde durch die Bromsulfaleinausscheidung im Magensaft erfaßt. Folgende Befunde ließen sich erheben: 1. Die entzündlichen Mucosaveränderungen verlaufen parallel zur Schwere des duodenogastrischen Refluxes. 2. Der durchschnittliche Säurereduktionsindex beträgt 87\%. 3. Der Billroth-I-Magen besitzt keine normale Motorik, sondern eine sogenannte Magenstumpfmotilität, die - wenn vorhanden - eine normale Entleerung ermöglicht.

Schliisselwörter: Billroth-I-Magen - Refluxgastritis - Restsekretion - Magenstumpfmotilität. 